\title{
A midwifery critical analysis of: A retrospective cohort study of the association between midwifery experience and perinatal mortality (Lawton et al., 2015)
}

\author{
Karen Guilliland ${ }^{A, B}$ MA, RM, RGON, ADN, MNZM • Lesley Dixon ${ }^{B}$ PhD, MA(Mid), BA (Hons), RM \\ - Claire MacDonald ${ }^{B}$ RM
}

${ }^{\mathrm{A}}$ Corresponding Author:nzcom@ nzcom.org.nz

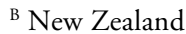
College of Midwives

\begin{abstract}
Background: New Zealand has a unique model of maternity care, with midwives as the predominant carers. A recently published retrospective cohort study sought to compare the experience levels of Lead Maternity Carer midwives with rates of perinatal mortality. The paper claimed that an association was found between first year of practice midwives and increased perinatal mortality. However, the study design was seriously flawed and the data do not support the conclusions that were drawn.

Aim: To systematically critique the study using the principles of epidemiological research.

Methods: The study design and methodology were critically analysed in terms of the three potential sources of error that occur in observational studies: bias (measurement error), confounding and chance. The potential impacts on results were considered.

Findings: Four areas of misclassification bias were identified, which introduced error to the data from the design stage. Important confounders were not distinguished and therefore neither excluded in the design stage nor adjusted for in the analysis. These flaws invalidate the results. The hypothesis test for the outcome of interest indicated an extremely high likelihood that the apparent association between midwives in their first year of practice and increased perinatal mortality was due to chance.

Conclusion: Because of the major methodological flaws in this research, no conclusions can be drawn from the results. This paper highlights the importance of involving members of the profession being studied in the research team to improve quality by providing crucial insight into the sector and identifying potential sources of error.
\end{abstract}

Key words: midwives' experience, first year of practice, perinatal mortality, critical analysis

\section{INTRODUCTION \& BACKGROUND}

The education and experience levels of midwives have been criticised in a recently published study by Lawton, Filoche, Geller, Garrett, and Stanley (2015). This paper claims that there is an association between increased perinatal mortality and Lead Maternity Carer (LMC) midwives who had less than one year's experience and did not have a previous nursing qualification (Lawton et al., 2015). The aim of our paper is to provide a comprehensive critical analysis of the study.

The hypothesis was that "pregnancies cared for by early career midwives would be associated with increased perinatal mortality compared with those cared for by midwives with 5-9 years of postmidwifery qualification experience" (p.2). The methodology used to test this hypothesis was a retrospective cohort study looking at routinely collected historical data linked to the Midwifery Council of New Zealand's (MCNZ) register of midwives to identify the year of midwifery registration, which was used as an indication of the experience level of the midwife providing care. The paper concludes that "pregnancies cared for by first year midwife-only LMCs were associated with a $33 \%$ increase in perinatal mortality" (p.6). Although the study stipulates that this is an association and causality cannot be claimed, it goes on to suggest a causal association between caregiver and perinatal mortality by stating
"The additional training that nurse-midwives receive could contribute to improved outcomes" (p.6).

Midwifery, as an autonomous health profession, welcomes robust and reliable research which can provide evidence to support and direct practice. Scientific research is a way of increasing knowledge through systematic inquiry, which commences with an hypothesis and then tests that conjecture using rigorous and reliable methods to answer questions related to the hypothesis (theoretical position) (Gavin, 2008). A study is designed to test the hypothesis against the null hypothesis, and whether the null hypothesis can be falsified (disproved), in other words, whether results indicating an association are more likely to be due to the hypothesis, than due to chance.

While this study presents a valid question, there are numerous flaws in the design and methodology used to test the hypothesis, which had the potential to influence the findings. The majority of the findings did not reach statistical significance, meaning that chance cannot be excluded. Using and linking large existing databases provides an inexpensive research method but can lead to the identification of statistical significance even when no association exists (Grimes, 2015). Hence caution is needed when interpreting results and any factors that may influence the results should be carefully considered. Instead of a cautious discussion 
about the data, its limitations and the uncertainty of the result, this study claims an association was found.

\section{THE METHODOLOGY OF THE PAPER}

The retrospective cohort study used record linkage to link routinely collected data on perinatal mortality rates with data on midwifery registration from the $\mathrm{MCNZ}$ register of midwives. The study included 233,215 births over the years 2005 to 2009 . The New Zealand Ministry of Health Information Group (MoHIG) provided the anonymised dataset of midwife registrations with identifiers of month/year of registration to the researchers. The researchers were able to map perinatal mortality outcomes to the LMC midwife with whom the woman booked during pregnancy. The primary outcome was perinatal mortality, including fetal deaths from 20 weeks gestation to neonatal deaths up to 27 days after birth. The exposure was years "of experience" (measured as years since registration: the significance of this distinction is outlined in the section on bias) according to eight categories, with each of the first five years as individual categories, followed by 5-9 years, 10-19 years and 20 years or more. Midwives were further stratified to "midwife-only", defined as midwives who did not have a prior nursing qualification, or "nurse-midwife", defined as midwives who had a prior nursing qualification. Women were analysed in two groups; "high risk" was defined as age $<21$ years or $>39$ years at the time of birth, multiple pregnancy, or parity of 4 or more; all other women were defined as "low risk". "High risk" women were excluded from the main analysis on this basis. Although data were presented on socioeconomic status/ deprivation quintile, ethnicity and mode of birth, these variables were not included in the analysis of perinatal mortality. The reference group was midwives with 5-9 years of experience, to which all other categories were compared.

\section{CRITIQUE OF THE METHODOLOGY}

When a researcher is setting up a research project, the methodology and research design need to be carefully considered so that any potential for bias or confounding is identified and measures are taken to minimise error and reduce the influence of confounders on the outcome of interest (Kirkwood \& Sterne, 2003).

\section{Misclassification bias}

Misclassification bias is a type of measurement error that epidemiologists usually aim to identify and minimise through good study design, as it cannot be controlled for in the analysis. It occurs when random and/or systematic errors occur in the way that people or groups are categorised. Our review found four areas in which misclassification bias occurred, which fundamentally flaws the research from the outset due to inherent errors in the data, thus nullifying the findings.

Firstly, the use of the MCNZ register of midwives to determine the experience levels of the midwife will have resulted in misclassification of midwives who qualified overseas. The register of midwives indicates the date of registration in New Zealand, not the date at which midwifery registration occurred in the country of origin. For example, a midwife who gained her nursing qualification in the United Kingdom in 1991 and her midwifery qualification in the United Kingdom in 1994, but arrived in New Zealand and registered as a midwife here in 2004 will be listed on the MCNZ website as registering with both qualifications in 2004. This midwife would appear to be a midwife in her second year of practice in the study in 2005 but, in fact, she has had 11 years of experience in midwifery practice.

During the time period of this study there was a midwifery shortage so an overseas recruitment drive led to between 100 and 200 midwives each year coming to New Zealand to practise. Overseas qualified midwives made up $36.6 \%$ of the midwifery workforce in 2009 (Ministry of Health, 2014). This means that more than a third of midwives in the cohort were potentially categorised into the wrong experience bracket.

Secondly, the MCNZ states that since 2004 they have not always recorded previous health qualifications of registered midwives (MCNZ, private correspondence, 2015), meaning that an unknown number of midwives with a previous nursing qualification will have been misclassified as "midwife-only LMCs" in the study.

Thirdly, New Zealand-educated midwives will have been misclassified: where they have registered with the MCNZ on graduation from their undergraduate midwifery degree but not taken up their annual practising certificate in the first year after qualifying. When they later enter the profession with a bridging course, the data collection method would have them categorised in a more experienced group when in fact, they have less than one year of experience.

The fourth area in which misclassification has occurred is in the carer at the time of perinatal mortality - the study assumes this is the same midwife who registered the woman at the start of pregnancy care but this is often not the case. In practice, care is often transferred to specialist hospital services and the original LMC midwife may cease involvement in the woman's care when new risk factors for perinatal mortality arise. The Section 88 Guidelines for Consultation with Obstetric and Related Medical Services (Ministry of Health, 2012) provides a comprehensive set of indications where referral to a specialist and hand-over of clinical responsibility are indicated. The rates of transfer have been investigated by the Perinatal and Maternal Mortality Review Committee (2015)(PMMRC). Their analysis of the LMC midwife at registration in pregnancy, compared with the LMC at birth, for all stillbirths and neonatal deaths, showed that in 2013, of 427 registrations, 320 were with a self-employed midwife. Of these 320 , only 145 were still under the care of a self-employed midwife at the time of birth, while 171 , or $53.4 \%$ (more than half) had been transferred to hospital care. The PMMRC report states that "The changes in caregiver from registration to birth in this context are likely to represent appropriate transfer of at-risk mothers for secondary or tertiary care" (p.79). The care provider is only one of a potential range of influences on maternity outcome.

This misclassification of the midwives' experience levels, and misclassification of caregiver at the time of birth, introduces error to the study from the design stage with an unpredictable impact on results that cannot be adjusted for at the analysis stage. This sort of measurement error results in erroneous conclusions due to erroneous data, also known colloquially as "garbage in, garbage out" (Grimes, 2015, p.2).

There were further issues in the study related to how women were stratified based on their risk status and the lack of adjustment for confounding.

\section{Risk Stratification}

The criteria for excluding "high-risk" cases were inadequate. This study only had four criteria for "high risk" classification: age $<21$ years at time of birth, age $>39$ years at the time of birth, multiple pregnancy, or parity of 4 or more. All other women were defined as "low risk". These criteria are arbitrary and do not represent accepted best practice within the sector. Most definitions of "low risk" will ensure the exclusion of a long list of pre-existing medical conditions as well as issues that may occur during pregnancy, such as gestational diabetes or pre-eclampsia. These conditions, among others, have not been distinguished. 


\section{Lack of adjustment for confounding}

Within maternity there are known confounders which need to be controlled for when considering perinatal mortality. The PMMRC (2015) has identified that congenital abnormality and prematurity are the leading causes of perinatal mortality, as well as other obstetric antecedents. If the study's aim was to identify whether the experience of the carer impacted on perinatal mortality, then eligibility criteria would be required, with exclusion of fetal and congenital abnormality, late termination of pregnancy and prematurity. This was not done - all perinatal mortalities were included in the dataset with no attempt made to adjust for, or exclude, non-viable pregnancies. Furthermore, maternal comorbidities and risk factors should have been adjusted for as potential confounders. These are identified by the PMMRC (2015) as:

- $\quad$ Body mass index greater than 25

- Nulliparity

- Antenatal smoking

- Late registration with an LMC

- Living in areas of high socioeconomic deprivation

- Specific ethnicity

Having several risk factors compounds the risk.

In their first year of practice LMC midwives may be more likely to have higher rates of women with the above risk factors. They are building their caseload and will have availability to take a much higher proportion of women who register later in pregnancy. Early antenatal care may be protective against perinatal mortality due to timely screening and lifestyle advice (Dixon et al., 2014). Women who register later in pregnancy are more likely to be living with other risk factors for perinatal mortality. Multiparous women may be more likely to return to a prior LMC, and so early-career midwives may have higher rates of nulliparous clients, who are at higher risk of perinatal mortality (PMMRC, 2015). This study fails to distinguish these variables, and, in fact, classes nulliparous women as low risk.

Each of these variables may be independently associated with the exposure (experience of midwives) and the outcome (perinatal mortality), and are not on the causal pathway between the exposure and outcome. Results are therefore highly likely to be confounded, falsely elevating the rate ratio for midwives in their first year of practice.

\section{RESULTS}

When an apparent association is found in a study, we need to consider whether the results could have occurred because of alternative explanations, namely bias, confounding or chance. Because the study failed to address the first two of these sources of uncertainty, we may disregard the results on these grounds alone. For completeness, however, we now consider whether the results could have been due to chance as well.

\section{Hypothesis testing}

Results are generally considered statistically significant if the $\mathrm{P}$ value is less than 0.05 , indicating a less than $5 \%$ probability that an effect is due to chance. The hypothesis test for trend according to years of experience resulted in a $P$ value of 0.031 , a statistically significant finding which would have inferred a need for more exploration if the results had been reliable.

However, the outcome of interest that was highlighted and discussed by the authors featured an extremely high $\mathrm{P}$ value, from which no firm conclusions should have been drawn. The rate ratio 1.33 for direct-entry midwives in their first year of practice compared to the reference group resulted in a $\mathrm{P}$ value of 0.329 , indicating an extremely high likelihood that the apparent association between exposure and outcome is due to chance. Furthermore, in such a large study the p-value may be considered more relevant than in a smaller study. The null hypothesis of no association cannot be ruled out; in other words the data do not support the hypothesis that experience levels of midwives are associated with increased perinatal mortality.

Assessing the probability that any apparent association could be due to chance is a fundamental tenet of epidemiological research, so it is surprising that the authors have disregarded what their analysis has clearly shown. They have identified the possibility of type I error (that an effect is not present) yet rejected it. We contend that type 1 error is highly likely to be present and that this study has not found a relationship between early career midwives and increased rates of perinatal mortality.

\section{Internal validity}

The internal validity is "the degree to which the results of a particular study are free from bias and confounding" (Webb \& Bain, 2011). As we have shown there are four major areas of misclassification bias which means that the outcome of interest is not analysed according to accurate exposure data, thus invalidating the results. Confounding is highly likely to be present, with none of the accepted risk factors for perinatal mortality controlled for in the design or adjusted for in the analysis. The internal validity of the study is therefore highly compromised and no conclusions can be drawn about associations between the exposure and outcome of interest.

\section{External validity}

External validity is the extent to which the results of a study can be generalised to the population. The data used in this research were a snapshot of an historical period relating to outcomes for 2005 to 2009 and as such do not represent the current system of midwifery education and graduate requirements. The discussion did not situate the study period in its historical context nor include information about the subsequent changes to undergraduate education and the significant amount of recent work that has been undertaken to strengthen the support for midwifery graduates. The study, therefore, has no external validity, meaning that it cannot be generalised to the current maternity system in New Zealand, as the system has changed since the study took place.

\section{DISCUSSION}

Observational studies are always subject to bias and confounding to a certain extent - these issues can only be completely avoided through randomisation and blinding. However, where this is not possible, observational studies are an important source of evidence when they are well designed and conducted. Robust research identifies potential sources of bias and ensures the study is designed appropriately to minimise these. Potential confounders are identified and either excluded from selection or adjusted for in the analysis. These clear steps were not taken in this study.

High quality research requires a careful and cautious desire to determine the truth based on all the available information (Litman, 2007). It requires judgement and honesty and a careful evaluation of the data, possible errors, limitations and contradictory evidence. Uncertainties need to be identified and caution should be used when drawing conclusions with avoidance of exaggerated claims and assumption of causation based on one type of evidence. Most researchers recommend further exploration of their theory so that there are multiple types of evidence to provide sufficient foundation for building that theory. When researchers use the wrong techniques, misclassify, misinterpret their results, report 
selective results and draw unjustified conclusions then this can be considered to be poor research (Altman, 1994). Poor research may be done purposely or through ignorance; either way Altman (1994) argues that it can be considered unethical, misleading and ultimately causes harm.

Ioannidis (2005) suggests most research claims are more likely to be false than true and that many are measures of existing or prevailing bias. He defines bias as: the combination of various design, data, analysis, and presentation factors that tend to produce research findings when they should not be produced (Ioannidis, 2005, p.3). Ways of addressing this bias are to support high standards and identify and curtail prejudice, and to interpret findings with caution.

\section{To achieve robust, high quality research there is a need to involve midwives as one of the key maternity health professionals on any multi- disciplinary research group exploring women's health and maternity care.}

Perinatal mortality is an area of great importance and justifiable public concern. Publicly funded research in this area could lead to improved outcomes if it is well designed and conducted. However this study has not contributed to the evidence base, and has not even been able to test the original hypothesis due to the flaws in the study design that we have outlined here.

This study illustrates the need for engagement with the profession being studied for their crucial insight into potential sources of error, which may not be obvious to researchers if they are not working clinically within the sector. There is an increasing number of New Zealand midwives who have masters and doctoral degrees. As such they have a comprehensive understanding of research methodology and a working knowledge and insight into maternity care provision. To achieve robust, high quality research there is a need to involve midwives as one of the key maternity health professionals on any multi-disciplinary research group exploring women's health and maternity care. Midwives can provide important insights into maternity care provision which can support clarity and reduce or avoid bias and miscomprehensions.

\section{CONCLUSION}

Essentially our analysis of this paper has revealed that no correlation was found between the exposure and outcome of interest. The hypothesis test indicated a high likelihood that any association was due to chance. Furthermore, there are several major flaws in the methodology, including misclassification bias and confounding that has not been controlled for. Finally, the authors suggest that their findings can be generalised to the present day; however the historical dataset was taken from a period that bears little resemblance to the present system. All of these factors indicate that both the internal and external validity of the study are compromised. Unfortunately, the discussion and conclusion of the paper in question claimed an association confirming the original hypothesis when the data did not indicate that it existed.

As evidence-based practitioners, midwives welcome research that is robust and contributes to our knowledge base. When evidence of benefit builds we adjust practice to ensure high quality maternity care for mothers and babies. This study does not provide evidence of association as claimed. It was poorly designed and lacks research credibility.

\section{REFERENCES}

Altman, D. (1994). The scandal of poor medical research. British Medical Journal, 308, 283-284.
Dixon, L., Andrews, A., Eddy, A., Guilliland, K., Hendry, C., \& Houston, J. (2014). Changing trends in pregnancy registration for New Zealand women. Journal of Primary Health Care, 6(4), 279-285

Gavin, H. (2008). Understanding Research Methods and Statistics in Psychology. London: Sage Publications Inc.

Grimes, D. (2015). Epidemiologic research with administrative databases: red herrings, false alarms and pseudo-epidemics. Human Reproduction, 30(8), 1749-1752.

Ioannidis, J. (2005). Why most published research findings are false. PLoS Med, 2(e1), 24

Kirkwood, B., \& Sterne, J. (2003). Essential Medical Statistics (2 ed.). Oxford: Blackwell Publishing.

Lawton, B., Geller, S., Garrett, S., \& Stanley, J. (2015). A retrospective cohort study of the association between midwifery experience and perinatal mortality. International Journal of Gynecology \& Obstetrics, In Press.

Litman, T. (2007). Evaluating Research Quality: Guidelines for Scholarship. Canada: Victoria Transport Policy Institute.

Ministry of Health. (2012). Guidelines for Consultation with Obstetric and Related Medical Services (Referral Guidelines). Wellington: Ministry of Health.

Ministry of Health. (2014). Health of the Health Workforce 2013 to 2014. Wellington: Ministry of Health.

Perinatal and Maternal Mortality Review Committee. (2015). Ninth Annual Report of the Perinatal and Maternal Mortality Review

Committee: Reporting mortality 2013. Wellington: Health Quality \& Safety Commission.

Webb, P., \& Bain, C. 2011. Essential Epidemiology: An Introduction for Students and Health Professionals (2nd ed). Cambridge: Cambridge University Press.

\section{Accepted for publication November 2015}

Guilliland, K., Dixon, L., MacDonald, C. A midwifery critical analysis of: A retrospective cohort study of the association between midwifery experience and perinatal mortality (Lawton et al., 2015). New Zealand College of Midwives Journal 51, 59-62. http://dx.doi.org/10.12784/ nzcomjnl51.2015.9.59-62

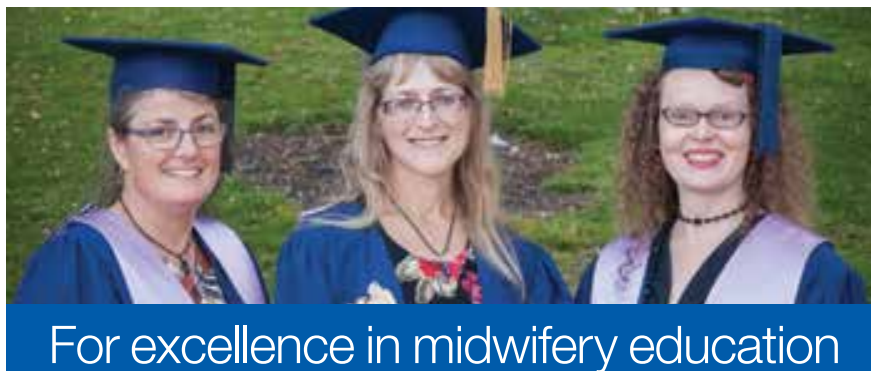

Our flexible, practice-focused programmes and courses are specially designed to meet the needs of midwives and the requirements of the Midwifery Council of NZ. We are offering the following online postgraduate papers in 2016:

2016

Feb 15-Apr 8 Sexually transmitted and other infections in pregnancy Feb 15-Apr 8 Preceptorship for midwives

Feb 15 - Jun 17 Knowledge and theory in midwifery

Feb 15- Jun 17 Midwifery assessment and decision-making

for complexity

May 2 - Jun 17 Politics and practice of breastfeeding

May 2 - Jun 17 Global midwifery and safe motherhood

July 4 - Aug 18 Maternal mental health

July 4 - Aug 18 Global midwifery: practical steps

July 4 - Oct 21 Research methods in midwifery practice

July 4 - Oct 21 Midwifery practicum for complexity*

Sep 5 - Oct 21 Promoting normal birth

Sep 5 - Oct 21 Maternal obesity

* The practicum includes a personal choice elective paper. If you wish to complete the complex care pathway including the practicum, please register your interest with Suzanne Miller (suzanne.miller@op.ac.nz) as places are limited.

\section{OTAGO}

